\title{
Las relaciones fiduciarias y sus contextos: continuidades, analogías y metáforas. (Presentación del número monográfico**
}

\author{
Fiduciary relationships in contexts: continuities, \\ analogies and metaphors. \\ (Presentation of the monographic issue)
}

JORDI MUNDÓ

\begin{abstract}
Resumen: Este número monográfico pretende contribuir a la reflexión sobre el interés de las relaciones fiduciarias en varios sentidos. En primer lugar, quiere coadyuvar a aclarar las ventajas y las limitaciones conceptuales y normativas del principio fiduciario para la reflexión filosófico-política y para la política práctica contemporáneas. En segundo lugar, aspira a enriquecer nuestra apreciación de los hilos entrecruzados de la constitución fiduciaria del mundo moderno mediante el examen de los desarrollos de esta idea en Europa y Estados Unidos. En tercer lugar, pretende refinar nuestra comprensión del potencial que tiene su aplicación a diversas áreas interconectadas de la filosofía, la política, la economía, la sociología y el derecho. Finalmente, quiere proponer discusiones histórico-conceptualmente informadas sobre la relación entre el republicanismo y los principios fiduciarios.

Palabras clave: relaciones fiduciarias, historia conceptual, republicanismo
\end{abstract}

\begin{abstract}
This monographic issue aims to contribute to the reflection on the interest of fiduciary relationships in several ways. Firstly, it wants to help clarify the conceptual and normative advantages and limitations of the fiduciary principle for contemporary philosophicalpolitical reflection and practical politics. Secondly, it aspires to enrich our appreciation of the interlocking threads of the fiduciary constitution of the modern world by examining the developments of this idea in Europe and the United States. Thirdly, it seeks to refine our understanding of the potential of its application to various interconnected areas of philosophy, politics, economics, sociology, and law. Finally, it is intended to propose historically and conceptually informed discussions on the relationship between republicanism and fiduciary principles. Keywords: fiduciary relationships, conceptual history, republicanism
\end{abstract}

Recibido: 08/07/2020. Aceptado: 09/07/2020. Cómo citar este articulo: Mundó, J. (2020). Las relaciones fiduciarias y sus contextos: continuidades, analogías y metáforas. (Presentación del número monográfico). Daimon. Revista Internacional de Filosofía, (81), 7-16. https://doi.org/10.6018/daimon.436291

* Este trabajo se ha desarrollado en el marco del proyecto de investigación PGC2018-094324-B-I00 (MCIU/AEI/ FEDER, UE), sobre "Libertad política, derechos de propiedad, bienes comunes y política pública entendidos como relaciones fiduciarias". Quiero agradecer al profesor Emilio Martínez Navarro su generoso apoyo para que este monográfico saliera adelante; un agradecimiento que hago extensivo a los revisores anónimos de los artículos. Finalmente, valga este monográfico como reconocimiento colectivo de la contribución fundamental que hizo el filósofo Antoni Domènech a la concepción fiduciaria de la tradición republicana.

** Profesor de la Universitat de Barcelona. Correo electrónico: jordimundo@ub.edu. 


\section{Introducción}

En los últimos años, la investigación sobre relaciones fiduciarias ha sido muy intensa. Lo ha sido en las disciplinas jurídicas, muy señaladamente en los ámbitos del derecho civil y mercantil, pero también en derecho constitucional y derecho administrativo. También en el campo de la teoría económica hay una larga tradición de indagación de las virtudes y limitaciones de este tipo de relaciones asimétricas, modelizadas como una estructura de principal/agente. Asimismo, estos distintos tipos de investigaciones han acreditado un enorme potencial analítico y normativo cuando se trasladan a la comprensión de la naturaleza de la vida política pública. De modo que el propósito de este número monográfico tiene mucho que ver con la exploración del amplio espectro de posibilidades filosóficopolíticas que se abre al adoptar una perspectiva basada en el principio fiduciario. Se trata de un ejercicio que pretende ser genuino, consistente en la puesta en común de análisis que ansían ser fértiles para ulteriores discusiones, pero que sobre todo quieren aportar buenas razones para la solución de problemas filosófico-políticos y político-sociales prácticos del mundo de hoy.

Las relaciones fiduciarias tienen en común varios aspectos: (1) surgen allí donde alguien (según el contexto, un agente, fiduciario o fideicomisario) tiene confiada la capacidad -un cierto tipo de poder administrativo- para actuar en nombre de otro (según el contexto, el principal, fiduciante o fideicomitente), (2) para un fin determinado y (3) siempre para el bien e intereses del principal, no del propio agente. Esta relación asimétrica suele comportar que el agente (4) tenga un margen de autoridad discrecional para perseguir los fines del principal, por lo que (5) es una relación inherentemente de confianza. (5) Puesto que el agente tiene poderes que escapan al control del principal, en una relación fiduciaria suelen articularse mecanismos de rendición de cuentas que limiten, embriden o disipen los incentivos que tiene el agente para actuar para su propio interés y no en el del principal. Por eso, (6) en varios tipos de relaciones fiduciarias el principal puede romper unilateralmente la relación sólo con alegar pérdida de confianza.

Sin embargo, la descripción de estos u otros rasgos compartidos por muchas relaciones fiduciarias puede crear una apariencia superficial de homogeneidad de las mismas. Bien al contrario, históricamente las relaciones fiduciarias han exhibido una gran flexibilidad para acomodarse a situaciones muy diversas, de modo que cristalizan en formas y contenidos bien distintos según el contex to histórico, el dominio (político, jurídico, económico o social) o la intencionalidad normativa: puede ocurrir que no haya sólo dos partes, sino tres (que, además del principal y de quien actúa en su nombre, también haya un beneficiario que sea distinto del principal); puede darse el caso de que esta relación no nazca de la voluntad del principal sino por motivos contextuales, o que se origine por mandatos jurídicos, e incluso las hay que surgen de decisiones unilaterales del fiduciario; cabe la posibilidad de que el principal retenga cierta capacidad de influencia o control sobre las decisiones del agente, o puede que aquél esté por completo a expensas de la discrecionalidad de este; puede ser que la relación se dé en un contexto de relaciones iusprivadas, o que se desarrolle en el ámbito iuspúblico; puede darse la circunstancia de que el principal y/o el beneficiario sean una sola persona, pero en ocasiones está constituido por muchas; $y$, por dejarlo en algún sitio, puede haber relaciones fiduciarias que nazcan del consentimiento y otras que no, como en el caso 
del vínculo paterno-filial. De modo que para la comprensión de las relaciones fiduciarias el contexto importa, y mucho, pues son relaciones que están histórica, jurisdiccional y normativamente indexadas.

\section{Continuidades, analogías y metáforas}

Esta complejidad queda patente cuando tratamos de trazar una genealogía de las relaciones fiduciarias en la vida política. Como atestiguan varios artículos del presente monográfico sobre relaciones fiduciarias, es pertinente preguntarse por la etiología de las relaciones fiduciarias de un sistema político democrático, y también resulta estimulante tratar de contrastar, por ejemplo, la hipótesis del origen iusprivado de la estructura fiduciaria subyacente al poder político instituido. Sin embargo, en esta empresa es preciso entender la naturaleza intensional (con $s$ ) de las relaciones fiduciarias, puesto que su alcance, significado y consecuencias pueden variar según el contexto. La tutela y el trust constituyen ejemplos bien ilustrativos de esta complejidad.

Encontramos antecedentes valiosos de relaciones fiduciarias en codificaciones hammurábicas, judías e islámicas, pero muy señaladamente en el derecho romano clásico (Frankel, 2011; Criddle, Miller y Sitkoff, 2019). Sin embargo, resulta más arduo establecer claras continuidades entre todas ellas. Por ejemplo, es común sostener que las relaciones fiduciarias se originaron en el derecho (privado) romano y que este esquema se aplicó por analogía a la comprensión de las relaciones entre los cargos públicos y la ciudadanía (Cicerón, 1928, I.85; Séneca, 1929, I.IV.2). En este sentido, resulta muy llamativo -a la vez que congruente- que Cicerón utilice la concepción legal abstracta de tutela para describir apropiadamente la relación entre cargos públicos y los ciudadanos que están bajo su jurisdicción. Según el derecho romano, los niños que aún no habían llegado a la pubertad (doce años para las niñas y catorce los niños) y las mujeres independientes (sui iuris) de cualquier edad requerían de un tutor. El paterfamilias podía nombrar, en caso de muerte prematura, a un tutor, que no necesariamente tenía que ser un pariente. En su origen, la tutela tenía como fin preservar la propiedad familiar, pero más adelante incluyó la obligación pública de procurar el bienestar de los menores. Al aplicar la noción de tutela al papel de los cargos públicos, es posible que Cicerón tuviera en cuenta diversos aspectos. Así, los creadores de la tutela del gobierno -quienes eligen a los cargos públicos-son a su vez sus tutelados. Los cargos públicos tienen la obligación de servirles y de rendir cuentas ante ellos -como también ocurre con la tutela iusprivada- para su bien. Entre las principales responsabilidades de los cargos públicos -análogas a las de la tutela- están las de velar por la seguridad y las posesiones de los tutelados. Y, a semejanza de lo que le ocurre al tutor a quien se ha confiado esa función por parte del paterfamilias, se confía a los cargos públicos la res publica o res populi. Pero, ¿hasta qué punto esta analogía precisa la naturaleza de la relación fiduciaria en el dominio iuspúblico? ¿En qué medida los tutelados -supuestamente, la ciudadanía- tienen a su alcance mecanismos de exigencia de rendición de cuentas? Una relación fiduciaria entraña siempre una tutela en sentido metafórico o requiere que esta deba tener un correlato jurídico con efectos prácticos previsibles?

La idea de la existencia de una relación de tutela la encontramos también en fuentes carolingias tempranas medievales, previas a la recepción del derecho romano, que descri- 
ben la función de la monarquía como algo equivalente a la tutela, de modo que el rey era concebido como un tutor regni (Ullmann, 1969, 177). Entonces, cabría pensar que hay un origen medieval de la tutela independiente del antecedente romano clásico, lo que nos llevaría a que no hubo un único predecesor de esta concepción. Sin embargo, resulta muy revelador constatar que el redescubrimiento del derecho romano y su aplicación al análisis de la monarquía medieval permitió un grado de precisión analítica que coadyuvó a superar las articulaciones meramente metafóricas de la tutela que predominaban hasta ese momento. En este sentido, para los juristas medievales, la analogía legal implicaba algo más, la técnica de la equiparatio, que permitía una fertilización cruzada de ideas, de modo que podían tratarse en términos equivalentes dos o más asuntos que a priori no pertenecían al mismo dominio y que aparentemente tenían poco en común (cf. Kantorowicz, 1957; 1984, 51-52). La historia de las relaciones fiduciarias está llena de ejemplos de estas fertilizaciones cruzadas para la búsqueda de soluciones no sólo teóricas, sino eminentemente prácticas, a nuevos problemas político-sociales.

Otro caso de interés para entender la lógica de las posibles continuidades y discontinuidades de la concepción nuclear de las relaciones fiduciarias tienen que ver con el trust inglés. Por lo pronto, la definición de trust no parece muy clara. En su ensayo fundamental sobre este asunto, Maitland $(1929,43)$ sostiene que no existe una definición estándar de trust, sino que hay muchas aproximaciones. Cuando para definir mejor los contornos onomasiológicos y semasiológicos del trust tratamos de recurrir a sus antecedentes, pronto descubrimos que se trata de una empresa llena de complicaciones. El trust inglés es un dispositivo legal único sobre cuyo origen existe un debate abierto (Maitland, 1929; Avisheh, 1996). De hecho, hay cuatro teorías principales sobre el origen del trust: la romana clásica (Blackstone, 1979, II, 328), la germánica (Holmes, 1899), la islámica (Maksidi, 1981; Schoenblum, 1999) y la de etiología romano-germánica, sostenida por Pollock y Maitland (1898). Hasta el siglo XIX, se consideraba que el trust no era sino una versión del fideicommissum romano clásico. Pero poco a poco ganó predicamento la tesis de que su origen radicaba en la ley sálica del Salmannus; y en fechas recientes ha ganado peso el argumento de que el trust estuvo muy marcado por influencias del waqf islámico (Avisheh, 1996).

Sea como fuere, parece acreditado que la extensión de la práctica del antecedente del trust, el use, radicó en que permitía sortear las rigideces del common law en punto a la posesión y a la transferencia de propiedad (Avisheh, 1996). El sistema de los uses se basaba en la constitución de una propiedad fiduciaria a través de una interposición de personas, con el fin de eludir obligaciones relacionadas con la herencia o con limitaciones sobre la transmisión de esa propiedad. Maitland (1929) sugiere que el primer empleo de los uses pudo darse en el siglo XIII, habilitando tierras para uso de los frailes franciscanos, cuya orden seguía normas que prohibían la posesión de propiedades, individualmente o como comunidad. Para contrarrestar los efectos negativos en la recaudación impositiva de una aplicación generalizada de los uses, se restringió su aplicación mediante el Statute of Uses de Enrique VIII en el año 1535. Sin embargo, continuaron reconociéndose excepciones, y es a partir de estas que evolucionó el sistema legal inglés del trust. De este modo, resultaría que el use inglés, el fideicomissum romano y el Salmannus sálico tuvieron, entre otras, una causa común: surgieron como resultado de insuficiencias en las leyes positivas en relación con la propiedad (Seipp, 2011). Para alguien entrenado en derecho continental resulta poco intuitivo -y, a menudo, fuente de 
confusión- descubrir que el common law no conoció el trust como dispositivo legal o como práctica, puesto que los trusts se hacían cumplir mediante los tribunales de equity, no mediante los de common law (Seipp, 2011). Sólo en el último siglo y medio los asuntos de common law y de equity se tratan de forma integrada. Por eso, sin una adecuada contextualización, puede carecer de sentido atribuir el origen moderno de las relaciones fiduciarias en la vida política a la inveterada práctica del trust inglés. Como va dicho, no basta con descubrir meras analogías o metáforas superficiales, sino que requiere rastrear e interpretar trayectorias históricas que pueden tener discontinuidades y fertilizaciones cruzadas.

En el propio universo del derecho fiduciario anglo-americano -distinto del inglés- ha habido reflexiones críticas sobre el uso excesivo de la analogía y la metáfora. Lo cual no es óbice para que varios de los autores que han realizado críticas certeras también sostengan que una concepción históricamente informada del derecho fiduciario ayuda a comprender que gran parte del potencial de adaptación de este a realidades cambiantes requiere de analogías, metáforas y fertilizaciones cruzadas que pueden acabar convirtiéndose en dispositivos jurídico-políticos vinculantes y normativamente plausibles (Frankel, 1983; DeMott, 1988; Massey, 1990; Scallen, 1993; Sunstein, 1993; Yeager, 2017).

\section{Investigaciones sobre las dimensiones del principio fiduciario}

La creciente preocupación de las últimas décadas por una concepción fiduciaria de la vida política puede deberse a motivos bien dispares, que van desde la búsqueda de una alternativa robusta a las limitaciones de las teorías contractualistas hasta una exploración de nuevas formas de lidiar con la corrupción política y el abuso de poder, pasando por la preocupación por ir más allá de una concepción puramente formal de la democracia. En este sentido, la recuperación de la idea de que la propiedad privada en último término está sujeta al bien público o que tiene una función social, de tal modo que no cabe concebir la propiedad como ilimitada sino que es mejor comprendida como un dispositivo fiduciario, abre nuevas vías analíticas y normativas sobre qué significa democratizar las relaciones de poder en las sociedades contemporáneas. Se trata de un asunto que en los últimos decenios ha involucrado también concepciones muy distintas acerca de los bienes comunes como superadoras de la mera distinción público/privado.

Este número monográfico pretende contribuir a la reflexión sobre el interés de las relaciones fiduciarias en varios sentidos. En primer lugar, quiere coadyuvar a aclarar las ventajas y las limitaciones conceptuales y normativas del principio fiduciario para la reflexión filosófico-política y para la política práctica. En segundo lugar, desea enriquecer nuestra apreciación de los hilos entrecruzados de la constitución fiduciaria del mundo moderno mediante el examen de los desarrollos de esta idea en Europa y Estados Unidos. En tercer lugar, pretende refinar nuestra comprensión del potencial que tiene su aplicación a diversas áreas interconectadas de la filosofía, la política, la economía, la sociología y el derecho. Finalmente, quiere proponer discusiones histórico-conceptualmente informadas sobre la relación entre el republicanismo y los principios fiduciarios.

El monográfico se abre con dos textos complementarios pertenecientes fundamentalmente (aunque no exclusivamente) al contexto histórico anglosajón de los siglos XVI y XVII. David Guerrero, en su artículo «Looking for democracy in fiduciary government. Historical notes 
on an unsettled relationship (ca. 1520-1650)», parte de la constatación de que una perspectiva reciente sobre los fundamentos normativos del derecho público ha propuesto concebir las relaciones entre ciudadanía y Estado como una relación fiduciaria, usando deberes fiduciarios del ámbito iusprivado para justificar limitaciones jurídicas y morales al poder del Estado. Asimismo, sostiene que el gobierno fiduciario también ha sido señalado como una característica distintiva del republicanismo y de la soberanía popular, puesto que sitúa a la comunidad política como fideicomitente y beneficiaria de cualquier acto administrativo. En su aportación, Guerrero revisa algunas concepciones protomodernas del gobierno considerando sus justificaciones explícitamente fiduciarias. Concluye con una interpretación fiduciaria del iusnaturalismo leveller, que considera especialmente necesario para entender $-\mathrm{y}$, a su juicio, que puede contribuir a restaurar- la relación del gobierno fiduciario con la democracia.

En continuidad histórico-conceptual con el texto de Guerrero, el trabajo de Jordi Mundó sobre «Poder político fiduciario y soberanía popular. Libertad política, confianza y revolución en la filosofía política de Locke» muestra cómo la concepción revolucionaria de la soberanía popular tuvo un eslabón fundamental en la filosofía política de John Locke, quien elaboró un argumento en favor de la libertad natural y de la autonomía de juicio de los individuos, y en contra de la sujeción natural y la alienación de la libertad política. Mundó hace hincapié en cómo Locke concibe la autoridad política como un poder político fiduciario instituido para el fin del bien público. Según esta concepción fiduciaria, cuando los gobernantes actúan para fines distintos de los encomendados, arbitrariamente o por su interés propio, la confianza se pierde y el ejercicio del poder político regresa a las manos del pueblo libre, afirmándose así la soberanía de este.

A estos dos estudios les siguen tres textos que tratan de la importancia del principio fiduciario en concepciones y prácticas en torno a la Revolución francesa. La historiadora de las Revoluciones francesa y de Santo Domingo/Haití, Florence Gauthier, en su trabajo «Relaciones fiduciarias, libertad política, derecho a la existencia. Soberanía popular y separación de poderes en la Revolución francesa (1789-1795)», argumenta sobre el modo en que, en 1789, la convocatoria de los Estados Generales despertó el entusiasmo del pueblo al convertir las "comunas" en el lugar de ejercicio de su soberanía, vinculándolo al sistema electoral de los agentes de confianza revocables. El estudio hace hincapié en que, en 1793-1794, la guerra civil, liderada por la aristocracia de los ricos contra la cultura política popular, no pudo impedir ni la redistribución de tierras ni el programa de economía política popular. Y culmina su reflexión señalando el modo en que la República montañesa de 1794, a pesar de su brevedad, aprobó una constitución comunal basada en las relaciones de confianza entre el pueblo y sus mandatarios, algo que, a su juicio, ha sido imposible olvidar.

Tanto Gauthier como Yannick Bosc, historiador de la Revolución francesa y especialista en Robespierre, arguyen en favor de la importancia del principio fiduciario y su conexión con el principio de soberanía popular. De modo explícito, Bosc, en la investigación que lleva por título «Représentants, mandataires et commettants: Robespierre, la relation fiduciaire et le droit à l'existence matériel et politique» sostiene que, durante la Revolución francesa, el trabajo político del "lado izquierdo", del cual Robespierre fue uno de los portavoces, consistió en aplicar los principios de la Declaración de los Derechos del Hombre y del Ciudadano. Esto implicaba, argumenta Bosc, que el pueblo soberano tenía la capacidad de controlar el ejercicio del poder fiduciario de sus agentes. La función de este dispositivo, concluye, es garantizar el derecho 
natural del hombre a la existencia, que es la condición principal de la libertad y la razón de ser de la república. El texto de Yannick Bosc destaca por su original aportación históricoconceptual sobre las nociones fiduciarias de représentants, mandataires y commettants.

En la misma línea, acreditando el interés y la oportunidad de la reconstrucción fiduciaria de la concepción política revolucionaria de Robespierre, Pablo Scotto, en su trabajo sobre «Soberanía popular y concepción fiduciaria de los representantes públicos en Maximilien Robespierre», muestra que, en su discurso del 10 de mayo de 1793 sobre la Constitución, Robespierre vincula una concepción fiduciaria de los representantes públicos con una defensa de las virtudes de la democracia, que entiende como el único sistema político en el que los gobernantes, al ser parte del pueblo, tienen los mismos intereses que este. A juicio de Scotto, la defensa de la soberanía popular, unida a la primacía del poder legislativo, constituyen el fundamento de su concepción de una economía política popular; y trata de evidenciar que solamente esta clase de economía es compatible con una República cuyo primer objetivo sea la garantía de los derechos naturales del hombre.

Tras las distintas experiencias y recorridos teóricos y conceptuales fiduciarios europeos, el monográfico cruza el océano Atlántico para ofrecer tres trabajos originales del contexto estadounidense, que conectan en muchos aspectos con los artículos precedentes. Cronológicamente, el primero es el artículo de Bru Laín, que lleva por título «Ni absoluta, ni exclusiva. Una reconstrucción de la concepción de la propiedad republicano-jeffersoniana». En el mismo, sostiene que el principio político-filosófico que subyace a la concepción de la propiedad republicano-jeffersoniana es el del pacto fiduciario. Para ello, Laín realiza una definición de los principios que, a su juicio, informan las relaciones fiduciarias para aplicarla a dos casos de estudio que considera que están estrechamente vinculados en el republicanismo de Jefferson: el gobierno político y la propiedad. Concluye que la concepción que denomina republicana-fiduciaria es la que alimenta el principio de "utilidad pública" de la propiedad privada, presente todavía hoy en el constitucionalismo democrático.

El asunto de la concepción de los derechos de propiedad y sus límites está explícitamente articulado en el trabajo del filósofo político francés Jean-Fabien Spitz, sobre un autor relativamente poco conocido en nuestro contexto, Thomas Skidmore. En su artículo, titulado «Thomas Skidmore et le droit de transmettre et d'hériter», Spitz nos presenta los argumentos de Skidmore, uno de los principales representantes del agrarismo en los Estados Unidos de la primera mitad del siglo XIX. Inspirado por las ideas desarrolladas por Thomas Paine en Agrarian Justice, Skidmore publicó en 1829 el libro The rights of Man to property, en el que desarrolla las consecuencias de la idea según la cual, siendo el mundo una propiedad común de todos los hombres, cada uno tiene un derecho imprescriptible a una parte igual de los recursos naturales. Entre estas consecuencias figura la tesis de que este derecho hace imposible el derecho a testar, pues tal derecho haría de todo punto inaplicable que cada nuevo individuo incorporado tuviera acceso a la justa parte de propiedad a la que tendría derecho. A juicio de Spitz, Skidmore elabora así una teoría precisa acerca de las razones por las que el testador, tras su muerte, no puede tener derecho alguno sobre los bienes de los que fue propietario en vida.

Concluye esta triple reflexión fiduciaria sobre la propiedad en el contexto estadounidense la investigación de Marcos de Armenteras Cabot sobre «La aplicación de la doctrina del public trust en Estados Unidos: de la protección de los bienes comunes a la conserva- 
ción del medio ambiente». El artículo desarrolla un argumento acerca de cómo, desde el siglo XIX hasta la actualidad, la doctrina del public trust ha jugado un papel determinante en la protección de los bienes comunes en Estados Unidos. Esta doctrina, que el autor considera heredera de la tradición jurídica romana, basa su estructura conceptual en una relación fideicomisaria entre agente y principal, y fue inicialmente invocada para la salvaguarda de los bienes comunes de su cercamiento y privatización. Marcos de Armenteras da cuenta de la evolución y aplicación actual del public trust, concluyendo que este instituto se ha ido transformado en un instrumento jurídico clave para la protección ambiental.

A la exploración del contexto estadounidense le siguen dos trabajos que reflexionan acerca de las relaciones fiduciarias en diálogo con la tradición socialista. El texto de Edgar Manjarín, sobre «Marx y la tradición iusnaturalista en un mundo industrializado», parte del supuesto de que la obra de Marx presenta una base normativa congruente con un núcleo de presupuestos procedentes de la tradición iusnaturalista. Manjarín argumenta que esta tradición de origen medieval partió de una revisión de la codificación tardía del derecho romano. Sin embargo, considera que la evolución conceptual de derechos naturales subjetivos culminó, en su forma más radical, con el impulso de la movilización popular democráticofraternal de la Revolución francesa. Pero, argumenta, para Marx la defensa de los derechos naturales no se correspondía con las condiciones objetivas. El artículo concluye que Marx realizó una reelaboración teórico-práctica en punto a sintetizar este núcleo normativo para la comprensión de las transformaciones económicas de un mundo industrializado, incorporándola así al programa político socialista.

La investigación de Julio Martínez-Cava, titulada «Enemigo a las puertas. La libertad política y los principios fiduciarios en el socialismo británico», se propone proporcionar algunas claves históricas y conceptuales para comprender la historia del socialismo británico libertarian y su relación con la concepción fiduciaria, tanto del poder político como del poder económico. Martínez-Cava sostiene que las expresiones de este socialismo no son homogéneas, que convivieron con otras ideas rivales -llegando en ocasiones a mezclarse con ellas- y que fueron formuladas siempre como respuestas concretas a los problemas que planteaba cada momento histórico. Su argumento desarrolla la idea de que, desde el socialismo republicano de algunos seguidores de Robert Owen hasta los desafíos que planteó la New Left, las teorías fiduciarias estuvieron presentes en los textos de estos socialistas libertarian.

A este extenso bloque de artículos sobre concepciones fiduciarias histórico-conceptualmente contextualizadas le sigue un trabajo de Adrián Herranz Herrer, de índole fundamentalmente teórico-conceptual, sobre «Democracia republicana y autoridad política fiduciaria». Herranz propone una justificación de la democracia, y de la autoridad política derivada de la misma, a partir del ideal republicano de libertad como no-dominación. Argumenta que los procedimientos democráticos tienen valor por sí mismos porque son mecanismos de decisión donde hay reciprocidad en la libertad. De este modo, ofrece una justificación no-instrumental y no-perfeccionista de la participación política, para continuar mostrando que la autoridad debe ser adecuadamente controlada para evitar que exista dominación, razón por la cual tiene que concebirse como una relación fiduciaria, en la que los gobernantes actúan como agentes de la ciudadanía. Concluye su análisis haciendo hincapié en la necesidad de que los gobernantes cumplan con sus deberes fiduciarios y que los ciudadanos desarrollen virtudes cívicas. 
El monográfico se cierra con dos investigaciones que giran alrededor de la propuesta de la renda básica. En la primera, que lleva por título «Renta Básica y Renta Máxima: una concepción republicano-democrática», María Julia Bertomeu y Daniel Raventós se proponen fundamentar y mostrar la viabilidad de una Renta Básica, que conciben como resguardo de un mínimum de existencia social para todos, que permitiría garantizar una universalización de la libertad republicana. Además, desarrollan una justificación de una renta máxima, entendiendo que la acumulación de riqueza y, por ello mismo, de poder económico y político de los muy ricos, atenta contra el deber fiduciario de las repúblicas democráticas de garantizar una "vida normal" a todos sus habitantes. En su argumento incorporan una reflexión sobre la concepción fiduciaria de los derechos humanos como dispositivo de universalización de los mismos.

Finalmente, en su trabajo sobre «Poder de negociación y distribución social de capacidades para la nacionalización de la vida económica: ¿por qué la renta básica es un proyecto democratizador?», David Casassas analiza de forma original el potencial del acceso incondicional a recursos en la conformación de relaciones productivas y reproductivas libres en cuatro tiempos. En primer lugar, defiende una noción de democracia (económica) que exige la presencia de recursos incondicionalmente predistribuidos. En segundo lugar, explora el vínculo entre predistribución y poder de negociación. En tercer lugar, presenta el derecho a la existencia como condición para una cooperación social efectiva que merezca la pena ser cuidada. Concluye su ensayo mediante la discusión del papel de la renta básica en procesos de lo que denomina "nacionalización de la vida económica", entendidos como expresión de la voluntad democrática de la ciudadanía.

\section{A modo de conclusión}

Más allá de los términos expresamente fiduciarios, en los artículos del monográfico puede hallarse un haz de conceptos recurrentes conectados con las relaciones fiduciarias: republicanismo, soberanía popular, iusnaturalismo, democracia, socialismo, revolución, economía política popular, libertad política, propiedad, autoridad, derechos humanos, renta básica. Recientemente se ha afirmado con acierto que "[t]he idea of fiduciary government is as timely as it is timeless" (Criddle et al., 2018, 1). Bien parecería que esto vale para otros muchos dominios fiduciarios, no sólo para el de la política pública. De modo que, si las investigaciones contenidas en el presente monográfico pueden contribuir en alguna medida a la actualización del potencial analítico y normativo de las relaciones fiduciarias y a la articulación de un diálogo franco entre distintas disciplinas que coadyuve a avanzar en la democratización real del mundo contemporáneo, este trabajo colectivo habrá cumplido su cometido.

\section{Referencias}

Avisheh, A. (1996), "The Origins of the Modern English Trust Revisited", Tulane Law Review, 70(4), pp. 1139-1164.

Blackstone, W. (1979), Commentaries of the Laws of England: A Facmisile of the First Edition of 1765-1769, ed. Stanley N. Katz, 4 vols., Chicago: University of Chicago Press. Cicero, M.T. (1928), De Officiis, trad. Walter Miller, London: William Heinemann. 
Criddle, E.J. et al. (2018), "Introduction", in: Criddle, E.J.; Fox-Decent, E.; Gold, A.S.; Kim, S.H.; Miller, P.B. (ed.), Fiduciary Government, Cambridge: Cambridge University Press, pp. 1-20.

Criddle, E.J., Miller, P.B. y Sitkoff, R.H. (2019), The Oxford Handbook of Fiduciary Law, Oxford: Oxford University Press.

DeMott, D. A. (1988), "Beyond metaphor: An analysis of fiduciary obligation", Duke Law Journal, 5, pp. 879-924.

Frankel, T. (1983), "Fiduciary Law", California Law Review, 71(3), pp. 795-836.

Frankel, T. (2011), Fiduciary Law, Oxford: Oxford University Press.

Holmes, O.W. (1899), "Law in Science and Science in Law", Harvard Law Review, 12(7), pp. 443-463.

Kantorowicz, E. (1957), The King's Two Bodies: A Study in Medieval Political Theology, Princeton, NJ: Princeton University Press.

Kantorowicz, E. (1984), Mourir pour la patrie, tr. L. Mayali y A. Schütz, Paris: Presses Universitaries de France.

Maitland, F.W. (1929 [1909]), Equity, Cambridge: Cambridge University Press.

Maksidi, G. (1981), The Rise of Colleges: Institutions of Learning in Islam and the West. Edinburgh: Edinburgh University Press.

Massey, C. (1990), “American fiduciary duty in an age of narcissism”, Saskatchewan Law Review, 54(1), pp. 101-120.

Pollock, F.; Maitland, F.W., (1898), The History of the English Law Before de Time of Edward I, 2nd. ed., Cambridge: Cambridge University Press.

Scallen, E. A. (1993), "Promises broken v. promises betrayed: Metaphor, analogy, and the new fiduciary principle", University of Illinois Law Review, 4, pp. 897-980.

Schoenblum, J. A. (1999), "The role of legal doctrine in the decline of the islamic waqf: comparison with the trust", Vanderbilt Journal of Transnational Law, 32(4), pp. 11911228.

Seipp, D. J. (2011), "Trust and fiduciary duty in the early common law", Boston University Law Review, 91(3), pp. 1011-1038.

Sèneca, L.A. (1926), Diàlegs a Serè; De la clemència, trad. C. Cardó, Barcelona: Fundació Bernat Metge.

Sunstein, C. R. (1993), “On analogical reasoning”, Harvard Law Review, 106(3), pp. 741791.

Ullmann, W. (1969), The Carolingian Renaissance and the Idea of Kingship, London: Methuen.

Yeager, D. (2017), "Fiduciary-isms: study of academic influence on the expansion of the law", Drake Law Review, 65(1), pp. 179-222. 